\title{
To Our Referees
}

We are grateful to those who helped us review papers in 1974-1975.

Ament, M.E., Los Angeles, Calif. (USA) Amer, M.S., Evansville, Ind. (USA) Amman, R.W., Zurich (Switzerland) Andersson, S., Stockholm (Sweden) Andre, C, Lyon (France) Arias, I.M., Bronx, N.Y. (USA) Aries, V., London (England) Audigier, J.C., Lyon (France) Avioli, L.V., St. Louis, Mo. (USA)

Baker, S.J., Tamil Nadu (India) Bank, S., Cape Town (South Africa) Baron, J.H., London (England) Bayless, T., Baltimore, Md. (USA) Belber, J.P., Martinez, Calif. (USA) Bengmark, S., Lund (Sweden) Benhamou, J.P., Paris (France) Benzi, G.M., Pavia (Italy) Bergel, F., Jersey (Channel Islands) Berlie, J., Paris (France) Berthelot, P., Paris (France) Billing, B.H., London (England) Bircher, J., Bern (Switzerland) Blair, E.L., Newcastle upon Tyne (England) Blomstrand, R., Stockholm (Sweden) Bloom, S.R., London (England) Blum, A.L., Zurich (Switzerland) Bode, Ch., Marburg a.d. Lahn (FRG) Bonjour, J.P., Bern (Switzerland) Borgstrom, B., Lund (Sweden) Bortoff, A., Syracuse, N.Y. (USA) Bouchier, I.A.D., Dundee (Scotland) Bouhours, J.F., Lyon (France) Bradley, S.E., New York, N.Y. (USA) Bralow, S.P., Philadelphia, Pa. (USA) Brasher, G.W., Temple, Tex. (USA) Brodie, D.A., North Chicago, Ill. (USA) Bronner, F., Farmington, Conn. (USA) Brooks, F., Philadelphia, Pa. (USA) Case, R.M., Newcastle upon Tyne (England) Charney, A.N., Washington, D.C. (USA) Chayvialle, J.A., Lyon (France) Chey, W.Y., Rochester, N.Y. (USA) Chou, C, East Lansing, Mich. (USA) Christensen, J., Iowa City, Iowa (USA) Christophe, J., Brussels (Belgium) Clarke, R.M., Nottingham (England) Clement, J., Dijon (France) Cocking, J.B., Ramsgate (England) Code, C.F., Los Angeles, Calif. (USA) Cohen, S., Philadelphia, Pa. (USA) Collan, Y., Helsinki (Finland) Condon, J.R., London (England) Conrad, M.E., Washington, D.C. (USA) Cooper, H.L., Salem, Mass. (USA) Cowley, D.J., Manchester (England) Creamer, B., London (England) Creutzfeldt, W., Göttingen (FRG) Csendes, A., Santiago (Chile)

Davenport, H.W., Ann Arbor, Mich. (USA) Dawson, A.M., London (England) Debas, H.T., Los Angeles, Calif. (USA) Decker, K., Freiburg i.Br. (FRG) Delcourt, A., Brussels (Belgium) Demole, M., Geneva (Switzerland) Deren, J.J., Philadelphia, Pa. (USA) Dhumeaux, D., Paris (France) Dinoso, V.P., Philadelphia, Pa. (USA) Dombal, P.T. de, Leeds (England) Doniach, D., London (England) Dowling, R.H., London Bridge (England) Drasar, B.S., London (England) Dreiling, D.A., New York, N.Y. (USA) Druckrey, H., Freiburg i.Br. (FRG) Duthie, H.L., Sheffield (England) Dyck, W.P., Temple, Tex. (USA)

Eastwood, M.A., Edinburgh (Scotland) Eiseman, B., Denver, Colo. (USA) Elder, J.B., Manchester (England) Ellis, F.H., Boston, Mass. (USA) Emas, S., Stockholm (Sweden) Erlinger, S., Paris (France)

Feldmann, G., Paris (France) Ferguson, A., Edinburgh (Scotland)

To Our Referees 
Fiasse, R., Leuven (Belgium) Figarella, C, Marseille (France) Fitzgerald, O., Dublin (Ireland) Fordtran, J., Dallas, Tex. (USA) Forell, M.M., Munich (FRG) Forsander, O.A., Helsinki (Finland) Foulk, W.T., Rochester, Minn. (USA)

Galambos, J.T., Atlanta, Ga. (USA) Gillespie, I.E., Manchester (England) Gjone, E., Oslo (Norway) Go, V.L.W., Rochester, Minn. (USA) Goebell, H., Essen (FRG) Goldberg, D.M., Toronto, Ont. (Canada) Gorbach, S.L., Boston, Mass. (USA) Graef, J. de, Brussels (Belgium) Grossman, M.I., Los Angeles, Calif. (USA) Gudman-Hథyer, E., Hellerup (Denmark) Guth, P.H., Los Angeles, Calif. (USA) Gutman, Y., Jerusalem (Israel)

Hadorn, B., Bern (Switzerland) Haenszel, W., Bethesda, Md. (USA) Hand, B., Ipswich (England) Hansky, J., Melbourne (Australia) Harper, A.A., Newcastle upon Tyne (England) Harrison, J.E., Toronto, Ont. (Canada) Hartroft, W.S., Honolulu, Hawaï (USA) Hase, T., Washington, D.C. (USA) Hendrix, T., Baltimore, Md. (USA) Hess, W., Zurich (Switzerland) Hill, M.J., London (England) Hirsch-Marie, H., Paris (France) Hofmann, A.F., Rochester, Minn. (USA) Holt, P., New York, N.Y. (USA) Hunt, J.N., London (England)

Imondi, A.R., Spring House, Pa. (USA) Isenberg, I., Los Angeles, Calif. (USA) Ishii, Y., Tokyo (Japan) Isselbacher, K.J., Boston, Mass. (USA)

Jacobson, E.D., Houston, Tex. (USA) Jagenburg, R., Göteborg (Sweden) Jarnum, S., Copenhagen (Denmark) Johnson, A.G., London (England) Johnson, L.R., Houston, Tex. (USA) Johnston, D., Leeds (England)

Kaess, H., Heidelberg (FRG) Kern, F., Denver, Colo. (USA) Kestens, P.J., Leuven (Belgium) Kirsner, J.B., Chicago, Ill. (USA) Klopper, P.J., Amsterdam (Netherlands) Konturek, S.J., Krakow (Poland) Kowalewski, K., Edmonton, Alta. (Canada) Kowlessar, O.D., Philadelphia, Pa. (USA) Kraft, S.C., Chicago, Ill. (USA) Krause, U., Uppsala (Sweden)

Landor, J.H., Piscataway, N.J. (USA) Leese, C.L., Sutton (England) Lev, R., New York, N.Y. (USA) Levine, R.A., Syracuse, N.Y. (USA) Lieber, C.S., Bronx, N.Y. (USA) Lin, M.T., Indianapolis, Ind. (USA) Lipkin, M., New York, N.Y. (USA) Lord, P.H., Beaconsfield (England) Lu, A.Y.H., Nutley, N.J. (USA) Lubran, M., Chicago, Ill. (USA) Luyckx, A., Leuven (Belgium) Mackay, I.R., Melbourne (Australia) Madge, D.S., Near Ashfort (England) Magee, D.F., Omaha, Nebr. (USA) Marchis Mouren, G., Marseille (France) Marks, I.N., Cape Town (South Africa) Martin, F., Dijon (France) Masson, P.L., Brussels (Belgium) Mendeloff, A.I., Baltimore, Md. (USA) Menguy, R., Rochester, N.Y. (USA) Mignon, M., Paris (France) Modigliani, R., Paris (France) Morson, B.C., London (England) Moshal, M.G., Durban (South Africa) Murray, T.M., Toronto, Ont. (Canada)

Nordstrom, C, Lund (Sweden)

Okuda, K., Chiba (Japan)

Palmer, E.D., Long Valley, N.J. (USA) Pansu, D., Lyon (France) Pascal, J.P., Toulouse (France) Paumgartner, G., Bern (Switzerland) Persson, G.G.A., Lund (Sweden) Pfeiffer, C.J., Newfoundland (Canada) Philips, S.F., Rochester, Minn. (USA) Picard, J., Paris (France) To Our Referees 196

Piper, D.W., St. Leonards (Australia) Pope, C.E., II, Seattle, Wash. (USA) Potet, F., Paris (France) Prosser, C.L., Urbana, Ill. (USA)

Ramwell, P.W., Shrewsbury, Mass. (USA)

Read, A.E., Bristol (England)

Rehfeld, J.F., Copenhagen (Denmark) 


\author{
Remmer, H., Tubingen (FRG) \\ Rey, J., Paris (France) \\ Reynolds, T.B., Los Angeles, Calif. (USA) \\ Rhodes, J., Cardiff (England) \\ Robert, A., Kalamazoo, Mich. (USA) \\ Rose, E.F., East London (South Africa) \\ Rosensweig, N.S., New York, N.Y. (USA) \\ Rothschild, M.A., New York, N.Y. (USA) \\ Roze, C, Paris (France) \\ Rubin, E., New York, N.Y. (USA) \\ Rudick, J., New York, N.Y. (USA) \\ Rune, S.J., Copenhagen (Denmark) \\ Said, S.I., Dallas, Tex. (USA) Samloff, M.I., Torrance, Calif. (USA) Sarles, H., Marseille \\ (France) Saunders, S.J., Cape Town (South Africa) Schaffner, F., New York, N.Y. (USA) \\ Schersten, T., Göteborg (Sweden) Scheuer, P.J., London (England) Schneyer, L.H., Birmingham, \\ Ala. (USA) Schoenfield, L.J., Los Angeles, Calif. (USA) Scratcherd, T., Sheffield (England) \\ Seidel, D., Heidelberg (FRG) Semb, L.S., Oslo (Norway) Shiner, M., London (England) Simon, \\ L., Jaszbereny (Hungary) Sircus, W., Edinburgh (Scotland) Siurala, M., Helsinki (Finland) \\ Skinner, D.B., Chicago, Ill. (USA) Small, D.M., Boston, Mass. (USA) Smyth, D.H., Sheffield \\ (England) \\ Soergel, K.H., Milwaukee, Wise. (USA) \\ Solcia, E., Pavia (Italy) \\ Spjut, H.J., Houston, Tex. (USA) \\ Stadil, F., Copenhagen (Denmark) \\ Summerskill, W.H.J., Rochester, Minn. (USA) \\ Taylor, W.H., Liverpool (England) Thjodleifsson, B., London (England) Thompson, J.C., \\ Galveston, Tex. (USA) Toner, P.G., Glasgow (Scotland) Turner, M.D., Rochester, N.Y. (USA) \\ Tuyns, A.J., Lyon (France) Tygstrup, N., Copenhagen (Denmark) Tytgat, G., Amsterdam (The \\ Netherlands) \\ Unger, R., Dallas, Tex. (USA) Urban, E., San Antonio, Tex. (USA) \\ Vantrappen, G., Leuven (Belgium) Venables, C.W., Newcastle upon Tyne \\ (England) Vilardell, F., Barcelona (Spain) Vl-achevic, Z.R., Richmond, Va. (USA) \\ Walsh, J.H., Los Angeles, Calif. (USA) Warshaw, A.L., Boston, Mass. (USA) Wasserman, R.H., \\ Ithaca, N.Y. (USA) Watkinson, G., Leeds (England) Watt, J., Liverpool (England) Webster, \\ P.D., Augusta, Ga. (USA) Wheeler, H.O., San Diego, Calif. (USA) Whitehead, R., Bedford Park \\ (Australia) Williams, J.A., Birmingham (England) Williams, R., London (England) Wilson, \\ D.E., Chicago, Ill. (USA) Winkler, K., Copenhagen (Denmark) Wormsley, K.G., Dundee \\ (Scotland) Worning, H, Glostrup/Copenhagen \\ (Denmark) Wynder, EX., New York, N.Y. (USA)
}

